\title{
Study on anti-jamming communication technology based on transform domain
}

\author{
Cai-Feng $\mathrm{Wu}$, Jia Yang and Yu-Fan Cheng \\ Shipbuilding Industry Corporation No.722 Institute, Wuhan, China \\ E-mail:wcf31415926@163.com
}

\begin{abstract}
Based on transform domain, this paper presents the research about anti-jamming communication technology. Firstly, it demonstrates the basic principle of transform domain communication system (TDCS), in which message is modulated depending on a kind of basic noise-like function to improve its concealment. Then, Based on twodimension time-frequency design, a communication system with transform domain and multiple users is proposed. By constructing sequence having perfect/quasi-perfect crosscorrelation characteristics, this system can eliminate or reduce the interference among multiple users. Finally, the simulation result indicates that this method can effectively solve the problems of active anti-jamming.
\end{abstract}

Keywords: Transform Domain; Two-Dimension of Time-Frequency; Anti-Jamming.

\section{Introduction}

The emission port and receiving port of transform domain communication system (TDCS) do spectrum sensing in the same time, marking interferences and avoiding the frequency slits which interferences belong to. Making use of only frequency slits which interferences haven't occupied to carry signal's power in emitting signal is a communication way of actively avoiding interferences.

A communication way is proposed to avoid interferences[1]. Taking advantage of local environment to extract spectrum information and reforming DSSS waveform. Radclioffe researched performance of TDCS with broad band, narrow band, single and multiple interferences and compared with DSSS, the result shows that, in bipolar modulation condition, the BER performance of TDCS is $12.8 \mathrm{~dB}$ better than DSSS[2]. Swackhammer analyzes and tells that TDCS has good performance of multi-user communication[3]. Non-Fourier domain TDCS expands transform domain to wavelet transform domain[4], wavelet packet transform domain[5]. TDCS can process with multiple transform 
domains. The ways of modulation can transmit data for cyclic code shift Keying(CCSK) and orthogonal coding(OC)[6].

This paper proposes the anti-jamming communication technology based on transform domain. Based on the environmental spectrum sensing and judgment, message is modulated depending on a kind of basic noise-like function to improve its concealment and make it difficult to be detected. And, based on twodimension time-frequency design, the interference among multiple users can be eliminated or reduced.

\section{Basic Principle of TDCS}

\subsection{Anti-jamming Characteristics}

In order to initiative avoid the spectrum of jamming signal, TDCS's emission port scans the EC environment with spectrum sensing module. The spectrum sensing module divides the signal into $\mathrm{N}$ subcarriers, then the spectrum judging module gets the availability of subcarriers depending on the preset threshold. In another word, when a subcarrier's power value is beyond the threshold, the system will consider this subcarrier is occupied and marks it with 0 , otherwise marks it with 1 . Therefore, the emission port will get a sequence $A=\left[A_{0}\right.$ $\left.A_{1} \ldots A_{k} \ldots A_{N-1}\right]$ consisted with those mark values from TDCS.

As Fig. 1 shows, random phase mapper generates a pseudo-random and multi-phase sequence $P=\left[e^{j m 0} e^{j m l} \ldots e^{j m k} \ldots e^{j m N-1}\right]$, then emission port makes sequence $\mathrm{P}$ times sequence $\mathrm{A}$ one by one to get the frequency domain expression $\mathrm{B}=\mathrm{A} * \mathrm{P}$ of fundamental modulation waveform(FMW).

$$
\begin{aligned}
& \mathrm{B}=\left[\begin{array}{llllll}
B_{0} & B_{1} & \ldots . . & B_{k} & \ldots . . & B_{N-1}
\end{array}\right] \\
& B_{k}=\lambda A_{k} e^{j m_{k}}, k \in\{0, \ldots \ldots . N-1\}
\end{aligned}
$$

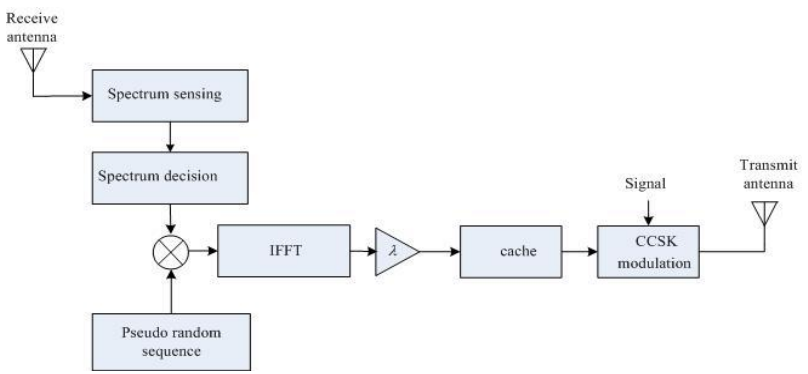

Fig. 1 TDCS Transmitter Block Diagram 
In which, $\lambda=\sqrt{N \varepsilon_{s} / N_{A}}$ is amplitude adjustment factor, $\varepsilon_{s}$ is the energy required to emit a symbol, $N_{A}$ is the number of mark 1 in utility spectrum sequence. $b=\left\{b_{0} b_{1} \ldots b_{k} \ldots b_{N-1}\right\}$ is the result of FFT of FMW. The basic function of TDCS can correctly reflect environment, its waveform has a similar characteristics with noise to hide the power of emission signal into background noises. This makes it hard to be effectively captured by monitors.

\subsection{Anti-jamming Characteristics}

Cycle code shift keying(CCSK) uses circular loop modulation to realize lowinterception transmission. Assume the interval of subcarriers is $\Delta f$, the number of mark 1 in utility spectrum sequence is $\mathrm{L}$, then dimension of signal is $\mathrm{N}=2 \mathrm{~L}$.

CCSK is similar with DSSS, according to the data provided by article ${ }^{[7]}$, with the length of recurrent code increases, detection probability of CCSK signal will be significantly reduced. So, for specific communication environment, in order to realize anti-intercept, it's better to choose a suitable length of recurrent code.

To achieve covert signal, obtaining random phase sequence of TDCS system by generating a chaotic sequence. Chaotic Sequence is an excellent pseudo-random sequence. It is easy to be generated in many ways. A pseudorandom sequence can be determined by mapping function, generating rules, and the initial conditions.

According to the way of generating a chaotic sequence, choosing a kind of mapping of chaotic iteration. The specific rules are as follows:

$$
x_{k+1}=f\left(x_{k}\right)=\left\{\begin{array}{cl}
b\left(1-u_{1} x_{k}^{2}\right) & -1<x_{k} \leq 0 \\
1-u_{2} x_{k} & 0<x_{k}<1
\end{array}\right.
$$

By setting the initial value, mapping parameters such as the number of system iterations to produce more random sequence. When the condition meets $x_{0}=0.82, u_{1}=1.8, u_{2}=2.0, b=0.85$, the chaotic sequence generated is as Fig.2 shows. In which the horizontal axis represents the number of iterations $\mathrm{k}$, the vertical axis is the mutative item $\mathrm{x}(\mathrm{k})$ of chaotic state space after the continuous iteration. What the Fig.2(a) shows is the chaotic mapping timing diagram while the initial value is 0.82 . The Fig.2(b) is the schematic diagram of sensitivity for mapping to iterative initial value (difference of initial values is $10^{-5}$ ). The result of Fig. 2 shows that: choosing any two initial values (difference of the two initial values is $10^{-5}$ ) while other parameters are same, two sequences is exactly same after 60 iterations. This shows that the iterative algorithm of chaotic sequence is highly sensitive to the initial value. Then, converting real-valued chaotic random sequence into binary random sequence. 


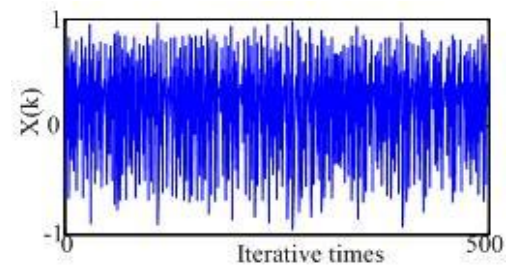

Fig. 2(a) Mapping of chaotic sequence

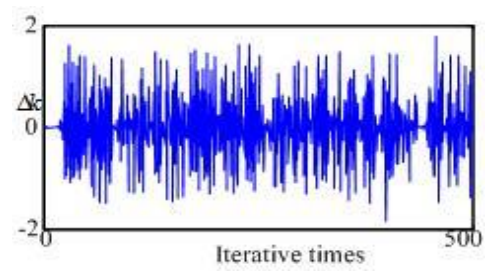

Fig. 2(b) Sensitivity of iterative initial value

Depending on the process of generating chaotic random sequence, we can freely set parameters as below:

- Iterative initial value $x_{0}$;

-Iterative mapping parameters $u_{1}, u_{2}, b$;

-the number of iteration;

-The length of binary random sequence.

Changing any one or more parameters will generate a separate set of pseudo-random sequence of multi-phase and TDCS basic function with different envelopes. Therefore, its changeable basic function makes it harder to be detected. So the anti-interception capability is improved.

\section{TDCS Based On Designing of Two-Dimension Time-Frequency}

This chapter proposes a design based on two-dimension of time-frequency. Set $A=\left[a_{0} a_{1} \ldots a_{L}\right]$ as a multi-phase sequence whose length is $\mathrm{L}$, this sequence owns auto correlation function $(\mathrm{ACF})$ :

$$
\varphi_{\mathbf{A}}(\tau)=\sum_{l=0}^{L-1} a_{l} a_{\bmod (l+\tau, L)}^{*}=\left\{\begin{array}{l}
L,(\tau=0) \\
0,(\tau \neq 0)
\end{array}\right.
$$

By two-dimension time-frequency design, depending on characteristics of correlated sequences, periodic CCF of sequence $\mathbf{c}^{(u)}$ and $\mathbf{c}^{(v)}$ is:

$$
\varphi_{\mathbf{c}^{(u)}, \mathbf{c}^{(v)}}(\tau)=\varphi_{\mathbf{A}}(l) \psi_{\mathbf{b}^{(u)}, \mathbf{b}^{(v)}}(m)+\varphi_{\mathbf{A}}(l+1) \psi_{\mathbf{b}^{(u)}, \mathbf{b}^{(v)}}(m-N)
$$

In which, $\tau=l N+m, 0 \leq l \leq L-1,0 \leq m \leq N-1$, and $\Psi_{u, v}{ }^{(\cdot)}$ is aperiodic CCF of $\mathrm{u}$ and $\mathrm{v}$, both of whose length is $\mathrm{N}$. as it shows with $\Psi_{\mathrm{u}, \mathrm{v}}(\tau)=$ $\sum_{\mathrm{i}=0}^{\mathrm{N}-1-\tau} \mathrm{u}_{\mathrm{i}} \mathrm{v}_{(\mathrm{i}+\tau)}^{*}$. When users whose number is $\mathrm{U}$ communicate in transform domain based on waveform modulation, every user takes $\mathrm{c}$ as basic function, takes $\mathrm{M}$ order circular code shift keying (CCSK) as modulation, number $\mathrm{U}$ user's emission signal $\mathbf{x}^{(u)}$ is generated by left-shifting $\mathbf{c}^{(u)} \tau_{u}$ bits $\tau_{\mathrm{u}} \in\left\{\begin{array}{llll}0 & 1 & \ldots \ldots & M-1\end{array}\right\}$ When each user transmit signals through a single path Rayleigh fading channel, number $\mathrm{v}$ user's receive signal $\mathrm{r}^{(v)}=\left[\begin{array}{llll}r_{0}^{(v)} & r_{1}^{(v)} & \ldots . . & r_{L N-1}^{(v)}\end{array}\right]$ can be expresses as 


$$
\mathbf{r}^{(v)}=\sum_{u=1}^{U} h_{u, v} \mathbf{x}^{(u)}+\mathbf{n}^{(v)}=\sum_{u=1}^{U} h_{u, v}\left\langle\mathbf{c}^{(u)}\right\rangle_{\tau_{u}}+\mathbf{n}^{(v)}
$$

Matching receive signal $\mathbf{r}^{(v)}$ and local reference receiver's signal with correlation calculation:

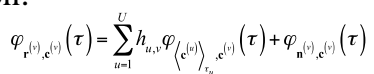

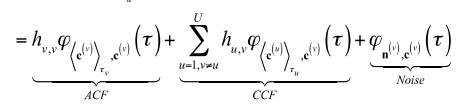

In Eq.(6), the first item is user v's periodic ACF of FMW after cyclic shifting $\tau_{v}$ and channel gain $\mathrm{h}_{\mathrm{v}, \mathrm{v}}$; The second item is the periodic CCF between $\mathrm{v}$-th user's local reference basis functions $\mathbf{c}^{(v)}$ and other users' receive signals; the third item is noise component.

For v-th user, only when $\tau_{u} \notin\left(-N+\tau_{v}, N+\tau_{v}\right)$, interference from the u-th user can be eliminated. Therefore, the necessary and sufficient condition to eliminate the interference from other users is:

$$
\tau_{v} \notin \bigcup_{u=1, u \neq v}^{U}\left(-N+\tau_{u}, N+\tau_{u}\right), \quad v=1,2, \cdots, U
$$

\section{Performance Analysis And Simulation}

This chapter is about system performance analysis and simulation. Fig.3 shows different bit error rates (BER) when $U=4$. Because of the non-orthogonality of spreading sequence, when noise figure (NF) increases, BER performances of MC-CDMA and traditional TDCS sharply decline. However, when $U=4$, $\mathrm{NF}=10 \mathrm{~dB}$, BER performance of anti MUI TDCS close performance single-user scenarios, which also verifies the ability of anti-interference of multi-user.

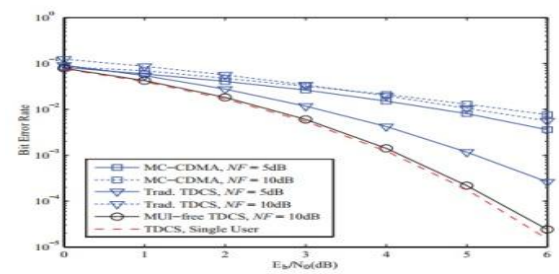

Fig. 3 BER performance for different systems in single fading channel 

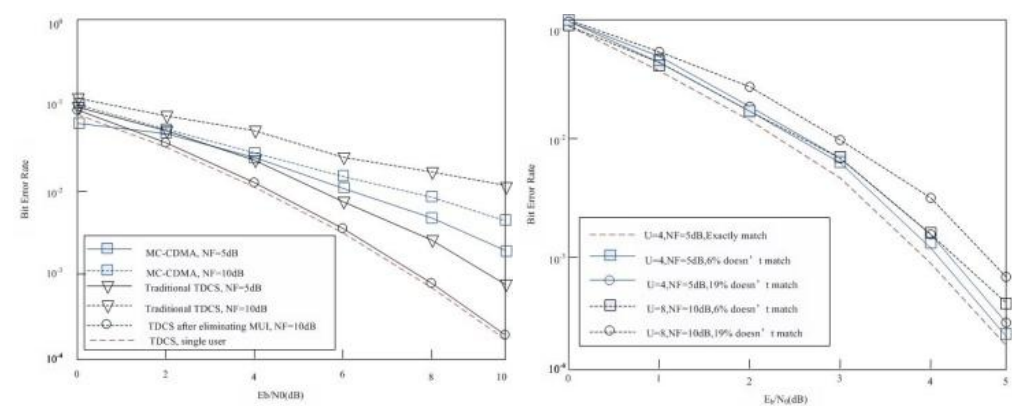

Fig. 4 BER of anti-MUI systems

Fig. 5 BER for different CRNs in multi-path fading channels

Fig.4 shows the error performance of the anti-MUI systems TDCS when the spectrum of perception does not match. For small number of users, as $U=4$, the error performance for each user gets closes to the ideal spectrum sensing performance. When $\mathrm{BER}=10^{-3}$, the value of $\mathrm{Eb} / \mathrm{N} 0$ only declines $0.15 \mathrm{~dB}$. However, for large number of users, as $U=8$, the BER performance significantly inferior to the ideal spectrum sensing. This is because when the spectrum sensing doesn't match, it no longer satisfies the restriction of Eq.(6)and can't avoid MUI, and remaining MUI reduces its BER performance.

Taking into account the multi-path fading channels, based on the BER performance of different random spreading, like channel COST207RAx6, taking long cyclic prefix to eliminate the influence of multi-path fading channels. MCCDMA and traditional TDCS takes minimum mean square error frequencydomain equalization (MMSE-FDE). The result is as Fig.5 shows. As it is known from the result, because of the nonzero periodic CCF between the transmitted signals, MC-CDMA and traditional TDCS are still affected by the MUI, but the anti-MUI TDCS obtains the BER performance closing to single-user scenarios BER performance.

\section{Conclusion}

This paper presents the basic principle of transform domain communication system (TDCS). The technology determines the free spectrum for transmission system TDCS by sensing and judging the environmental spectrum. And emitting information detected by modulating depended on noise-like basic function to effectively hide the interception of communications and low signal's interference. What's more, it proposes a multi-user communication system based on submarine transform domain, effectively solves the multi-user interference by taking advantage of perfect (or near perfect) sequence with two-dimensional 
design. The simulation results show that, TDCS can effectively achieve the technical characteristics of active anti-jamming.

\section{References}

1. E.H. German Transform Domain Signal Processing Study Final Report. Tech, rep., Reisterstown, MD: Contract: Air Force F30602-86-C-0133, DTIC: ADB132635,Aug.1988.

2. Radcliffe, Rodney A., "Design and Simulation of a Transform Domain Communication System, "MS Thesis, AFITI/GE/IENGI/96D-16, Air Force Institute of Technology, Wright-Patterson AFB OH, Dec 1996.

3. Swackhammer, Patrick J, "Design and Simulation of a Multiple Access Transform Domain Communication System" MS Thesis AFIT/GE/IENGI/99M-28,Air Force Institute of Technology, WrightPatterson AFB OH, Mar 1999.

4. Klein R.W, Temple, M.A, Claypoole, R.L, Jr, Raines, R.A, Stephens, J.P, "Wavelet Domain Communication system (WDCS) interference avoidance capabilities: analytic, modeling and simulation results," Military Communications Conference, 2001. MILCOM 2001. Communications for Network-Centric Operations: Creating the Information Force. IEEE Volume 2, Oct 2001:1034-1038.

5. Marion J. Lee, Michael A. Temple, Roger L. Claypoole, Jr., Richard A. Rains, "Transform Domain Communications And Interference Avoidance Using Wavelet Packet Decomposition," Wireless Communications and Networking Conference, 2002. WCNC2002. 2002 IEEE Volume 1, March 2002:255-259.

6. Sahai A, Hoven N, Tandra R. Some fundamental limits in cognitive radio [J]. Allenton Conf. on Common. Control and Computing 2004.

7. G.M. Dillard, M. Reuter, J. Zeidler, and et al, Cyclic Code Shift Keying: a Low Probability of Intercept Communication Technique, IEEE Transactions on Aerospace and Electronic Systems, July 2003, Vol.39:786-798. 